\title{
Outcomes of manual small incision cataract surgery (mSICS) compared with phacoemulsification from population based outreach eye camp, in Yogyakarta and Southern Central Java Region, Indonesia
}

\author{
Indra Tri Mahayana, ${ }^{1,{ }^{*}}$ Reny Setyowati, ${ }^{1}$ Tri Winarti, ${ }^{1,2}$ Suhardjo Pawiroranu ${ }^{1,3}$ \\ ${ }^{1}$ Department of Ophthalmology, Faculty of Medicine, Public Health, and Nursing, \\ Universitas Gadjah Mada, Yogyakarta, Indonesia \\ ${ }^{2}$ Universitas Gadjah Mada Academic Hospital, Yogyakarta, Indonesia \\ ${ }^{3}$ Dr Yap Eye Hospital, Yogyakarta, Indonesia
}

Submitted: 10 July 2018

Revised: 23 October 2018 Accepted: 6 November 2018

\begin{abstract}
In this study, we compared the effectiveness (comparison of post-operative visual acuity/VA) of phacoemulsification by ophthalmologists versus manual small incision cataract surgery (mSICS) by residents in a mass cataract surgery setting. This research was a cross-sectional study of 1137 cataract patients who underwent cataract surgery by ophthalmologists and residents in outreach eye camps during 2015-2017 ( 3 years). There were 554 patients who underwent phacoemulsification by ophthalmologists and 583 patients who underwent mSICS by residents. Basic patient characteristics data, such as age, sex and presurgical VA were recorded and we compared pre- versus post-operative VA (best corrected VA/BCVA) and surgical adverse events in 4 weeks post-operative follow-up. In basic subject characteristics, there were no differences in age and sex between the 2 groups, in which 602 (52.9\%) were men and 535 (47.1\%) were women. Overall 583 (51.3\%) eyes received mSICS and 554 (48.7\%) eyes received phacoemulsification. Visual acuity improvement $(\geq 6 / 18$ ) was achieved in $59.6 \%$ eyes after phacoemulsification and $53.5 \%$ eyes after mSICS. There were no statistical differences in visual outcome results between both groups $(p=$ 0.10). Severe surgical adverse events (nucleus drop and endophthalmitis) were found in 3 cases (0.3\%) and choroidal bleeding in 1 eye (0.1\%). The effectiveness of phacoemulsification and mSICS in improving visual acuity was found similar. The mSICS should be considered for more frequent use in high volume mass cataract surgery.
\end{abstract}

KEYWORDS cataract surgery; phacoemulsification; manual small incision cataract surgery; outreach program; community ophthalmology

\section{Introduction}

Worldwide, approximately 18 million people were bilaterally blind from cataracts. ${ }^{1}$ Cataract blindness is also the most significant cause of reversible blindness in Indonesia. There are an estimated 1.5 million visually impaired people with $1.5 \%$ of blindness in Indonesia as a result of cataracts, which is high compared to other South East Asian

countries. ${ }^{2}$ National survey in 2014 reported that cataract prevalence is highest compared to corneal or posterior segment disease, and glaucoma, thus affecting the quality of life and also socio-economic aspects of the patients. Due to Indonesia's tropical climate, cataracts tend to happen 15 years earlier than people in other subtropical countries. ${ }^{3}$

*Corresponding author: Indra Tri Mahayana

Department of Ophthalmology, Faculty of Medicine, Public Health, and Nursing, Universitas Gadjah Mada - Dr. Sardjito General Hospital, Jl. Farmako, Sekip Utara, Yogyakarta 55281, Indonesia

E-mail: tri.mahayana@gmail.com 
Cataract surgery results in almost immediate visual rehabilitation with minimal post-operative refractive-errors that reduce the number of disability adjusted life years (DALYs) caused by vision impairment. ${ }^{4} \mathrm{~A}$ well-managed eye unit with adequate support staff and infrastructure is able to undertake high-quality and high-volume surgery (1000-2000 or more operations a year). ${ }^{2}$ Outreach eye camps in rural areas is one of strategies to decrease the number of cataract backlog in the community. As the number of cataracts are increasing, community-based cataract outreach program is considered to be an important effort to relieve the associated health burden. There are some influencing factors in conducting 'eye camps', such as age distribution in a community, thresholds or indications used for surgery, and number of eligible patients who actually can receive surgery. ${ }^{5}$

Both manual small incision cataract surgery (mSICS) and phacoemulsification have become popular and common cataract surgical technique, in which phacoemulsification is the primary method for cataract surgery in developed countries. However, phacoemulsification is associated with high cost and maintenance demands of the equipment, therefore, significant efforts were made in developing countries to make more affordable cataract surgery. ${ }^{6}$ The mSICS has become considered as an alternative surgery for phacoemulsification, mostly in developing countries. The mSICS is a surgical technique which is possible in suboptimal conditions and serves to avoid the high maintenance cost of phacoemulsification, since it is faster and more cost effective than phacoemulsification. ${ }^{7}$

However, several comparable previous studies in Nepal have shown similar visual outcomes from both mSICS and phacoemulsification but there are no studies yet in Indonesia that report the significance of each visual outcome after the surgery. ${ }^{8}$ Similar studies in Egypt also stated that there were insignificant results of uncorrected visual acuity (UVCA) and best corrected visual acuity (BCVA) of patients who underwent phacoemulsification versus mSICS. ${ }^{7,9}$ In the Department of Ophthalmology, Faculty of Medicine, Public Health and Nursing, Universitas
Gadjah Mada, Yogyakarta, Indonesia, outreach cataract programs have been conducted frequently with approximately 15-20 programs annually. This study investigated the visual outcome of patients undergoing both phacoemulsification and mSICS in outreach programs, and analyzed common complications among patients with poor visual outcome after cataract surgery.

\section{Methods}

This research was a cross-sectional study of 1137 cataract patients who underwent cataract surgery by ophthalmologists and ophthalmology residents in outreach eye camps during 2015-2017 (3 years). There were 554 patients who underwent phacoemulsification by ophthalmologists and 583 patients who underwent mSICS by ophthalmology residents. This study was approved by the Medical and Health Research Ethics Committee of the Faculty of Medicine, Public Health, and Nursing, Universitas Gadjah Mada, Yogyakarta.

Phacoemulsification is a cataract removal technique using an ultrasound-based machine and micro-surgical instruments and involves temporal 2.5-3.0 $\mathrm{mm}$ sclerocorneal incision, followed by separate corneal port(s). ${ }^{10}$ Continuous curvilinear capsulorhexis (trypan blue-assisted capsulothexis is frequently conducted) is created and then hydrodissection is performed below the anterior capsule rim. ${ }^{11}$ The mSICS is a technique of extracapsular extraction in which the cataract nucleus is prolapsed from the capsular bag (with Sinskey Hook or hydrodissection injection) and extracted through a $6 \mathrm{~mm}$ scleral tunnel by irrigating vectis. ${ }^{10-12}$

Prior to surgery, visual acuity was recorded and diagnosis of cataract was made by slitlamp biomicroscopy anterior segment examination. The inclusion criteria were mature and immature senile cataract (Burrato's grade 3-4 cataract opacity), no systemic comorbidities, and gave informed consent. Subjects were excluded from the study if there were comorbidities that might complicate the surgery under local anesthesia, patients with co-morbidities that can complicate surgery (e.g., infection of the cornea), and loss of follow up at 28 days post-surgery. After the surgery, the subject was observed on Day 2, 5 and 28, then best corrected 
visual acuity (BCVA), complications, and findings were recorded.

Statistical analyses used in this study were independent sample t-tests to determine means between two independent groups and chi square $\left(x^{2}\right)$ tests to determine proportions.

\section{Results}

There were 602 (52.9\%) men and 535 (47.1\%) women involved in this study with no difference in age and gender between the two groups. Overall $583(51.3 \%)$ cataract surgeries were done with mSICS and 554 (48.7\%) with phacoemulsification. Visual acuity improvement $(\geq 6 / 18)$ was achieved in $59.6 \%$ eyes after phacoemulsification and $53.5 \%$ eyes after mSICS. There were no statistical differences of visual outcome (UVCA at D+28) results between both groups $(p=0.162)$ and proportion of normal vision between the two groups ( $p=0.233$; RR 0.897; 95\% Cl 0.75-1.074; Table 1).

The most common complication during surgery was aphakia which was found in $2.7 \%$ after mSICS and $0.7 \%$ after phacoemulsification. IOL drop and nucleus drop were found in $0.2 \%$ each after $\mathrm{mSICS}$, compared with $0.2 \%$ and $0.5 \%$, respectively in phacoemulsification. Pupil rhexis, dialysis, updrawn were the next most frequent intraoperative complications in $\mathrm{mSICS}(0.5 \%)$. But we found no significant differences in each complication between the two groups ( $p=0.375$, Table 2 ).

Severe surgical adverse events such as persistent corneal edema after surgery were found in 9 cases $(1.7 \%)$ after mSICS and 5 cases $(1,0 \%)$ after phacoemulsification. The number of posterior capsular opacification (PCO) after surgery were 4 cases $(0.7 \%)$ after mSICS and 1 case $(0.2 \%)$ after phacoemulsification. There were no significant associations found between postoperative complications and method used for cataract surgery ( $p=0.611$, Table 3$)$.

Table 4 shows the pre-existing ophtalmic diseases of the patients. Vitreous haemorrhage (1.9\%) and age-related macular degeneration (AMD) (1.9\%) were the most common pre-existing ophthalmic disease, followed by optic atrophy (1.5\%) and primary open angle glaucoma (POAG) (1.5\%).

Table 1. Visual acuity outcome of mSICS and phacoemulsification

\begin{tabular}{lccc}
\hline & mSICS & Phacoemulsification & $\boldsymbol{p}$ (RR; Cl 95) \\
\hline Visual acuity (logMAR), mean \pm SD & & & \\
Pre-surgery & $2.472 \pm 0.323$ & $1.627 \pm 0.393$ & $<0.001$ \\
& $(\sim 1 / 300)$ & $(\sim 1 / 60)$ & \\
Post-surgery (UCVA, D+28) & $0.707 \pm 0.615$ & $0.607 \pm 0.589$ & 0.162 \\
& $(\sim 6 / 30)$ & $(\sim 6 / 20)$ & \\
Normal vision ( $\leq 6 / 18)$ at D+28, $\%$ & 45.5 & 52.4 & $0.233(0.897,0.750-1.074)$ \\
\hline
\end{tabular}

logMAR: log minimum angle of resolution, RR: relative risk, Cl: confidence interval, UCVA : uncorrected visual acuity, $D+28: 28$ days post-surgery. Visual acuity pre- and post-surgery was statiscally different.

Table 2. Intra-operative complications

\begin{tabular}{lccc}
\hline & mSICS & Phacoemulsification & $p$ \\
\hline Pupil rhexis, dialysis, and up-drawn, $\mathrm{n}(\%)$ & $2(0.5)$ & $0(0.0)$ & 0.375 \\
Aphakia or PCR, $\mathrm{n}(\%)$ & $15(2.7)$ & $4(0.7)$ & \\
Nucleus drop, $\mathrm{n}(\%)$ & $1(0.2)$ & $1(0.2)$ & \\
IOL drop, $\mathrm{n}(\%)$ & $1(0.2)$ & $3(0.5)$ & \\
Hyphemia, $\mathrm{n}(\%)$ & $1(0.2)$ & $0(0.0)$ & \\
\hline
\end{tabular}

PCR: posterior capsule rupture, IOL: intra-ocular lens. 
Table 3. Post-operative complications

\begin{tabular}{llcc}
\hline & mSICS & Phacoemulsification & $\boldsymbol{p}$ \\
\hline PCO, $\mathrm{n}(\%)$ & $4(0.7)$ & $1(0.2)$ & 0.611 \\
Persistent corneal edema, $\mathrm{n}(\%)$ & $9(1.7)$ & $5(1.0)$ & \\
Endophthalmitis, $\mathrm{n}(\%)$ & $1(0.2)$ & $0(0.0)$ & \\
IOL decenteration, $\mathrm{n}(\%)$ & $1(0.2)$ & $1(0.2)$ & \\
Uveitis, $\mathrm{n}(\%)$ & $0(0.0)$ & $1(0.2)$ & \\
\hline
\end{tabular}

PCO: posterior capsular opacification, IOL: intra-ocular lens.

Table 4. Pre-existing ophthalmic diseases

\begin{tabular}{lc}
\hline Conditions & $\mathbf{n}(\%)$ \\
\hline Vitreous hemorrhage & $10(1.9)$ \\
AMD & $10(1.9)$ \\
Optic atrophy & $8(1.5)$ \\
POAG & $8(1.5)$ \\
Pathological myopia & $3(0.5)$ \\
Diabetic retinopathy & $3(0.5)$ \\
\hline
\end{tabular}

AMD: age-related macular degeneration, POAG: primary open angle glaucoma.

\section{Discussion}

In a community services activity, surgical options that are faster, more secure, and give a good visual outcome would be more preferred. One previous study revealed that in large numbers of cataract cases and in limited resource settings, such as in Africa, SICS is a more preferred technique than phacoemulsification. ${ }^{9}$ In the present study, mSICS and phacoemulsification surgery has the same effect in the treatment of cataracts. Phacoemulsification is not the only surgical option for best treatment effect. The two procedures provide similar visual results $(45.5 \%$ and $52.4 \%$ of patients in mSICS and phacoemulsification, respectively, reached BCVA $6 / 18$ or better at the end of follow-up) where the mSICS technique is less expensive, and less in need of technology as in phacoemulsification.

Cataract surgery dramatically increase patients' quality of life and markedly improve visual function. ${ }^{13}$ Although, phacoemulsification is the preferred method for cataract surgery worldwide, it is not always available in developing countries. ${ }^{14}$ The
mSICS is less expensive, less technology dependent and might be faster than phacoemulsification. Ruit et al. ${ }^{7}$ revealed that manual SICS is faster than phacoemulsification for advanced cataracts cases. Devendra et al. ${ }^{13}$ found that the average operative time in the phaco group was 16 min versus 10 $\mathrm{min}$ in the SICS group. Gogate et al. ${ }^{15}$ showed that a shorter duration of SICS decrease backlog of cataract patients.

Ruit et al. ${ }^{7}$ also indicated that manual SICS is far less expensive to perform than phacoemulsification due to phacoemulsification machine associated with consumables components (phacoemulsification tips, sleeves, and tubing) and ongoing maintenance. In mSICS, the operating microscope is the only expensive equipment. Gogate et al. ${ }^{15}$ revealed that mSICS was almost half the cost of phacoemulsification with easier learning curves. Moreover, Singh et al..$^{8}$ showed that sterilization procedures for $\mathrm{mSICS}$ is more available and inexpensive than phacoemulsification.

The mSICS is preferred option by surgeons and could be performed in rural and semi-urban settings and in eye-camps. ${ }^{15,16} \mathrm{It}$ is preferred in high volume outreach camp, such as in low socio-economic communities in the developing countries, due to its cost effective, comparable visual outcome, while being non machine dependent. ${ }^{16}$ The limitation of the present study was the best corrected visual acuity examination was heavily influenced by the condition of the posterior segment. Also, the operator was not a single surgeon. Operators of most of the mSICS were residents/ trainees with varying levels of expertise. 


\section{Conclusion}

The effectiveness of phacoemulsification and mSICS in improving visual acuity was found similar. The mSICS should be considered more in high volume mass cataract surgery.

\section{Acknowledgments}

There are no conflicts of interest to disclose, this research was made purely for developing better health community.

\section{References}

1. Ruit S, Tabin G, Chang D, Bajracharya L, Kline DC, Richheimer W, et al. A Prospective randomized clinical trial of phacoemulsification vs manual sutureless small-incision extracapsular cataract surgery in Nepal. Am J Ophthalmol. 2007;143(1):32-8.

2. Gogate P, Optom JJ, Deshpande S, Naidoo K. Meta-analysis to compare the safety and efficacy of manual small incision cataract surgery and phacoemulsification. Middle East Afr J Ophthalmol. 2015;22(3):362.

3. Wang W, Yan W, Fotis K, Prasad NM, Lansingh VC, Taylor HR, et al. Cataract surgical rate and socioeconomics: a global study. Investig Ophthalmol Vis Sci. 2016;57(14):5872-81.

4. Pan American Health Organization. Plan of action for the prevention of blindness and visual impairment. Proceedings of the 53rd Directing Council of PAHO, 66th Session of the Regional Committee of WHO for the Americas. Washington DC, USA: PAHO. 2014.

5. Batlle JF, Lansingh VC, Silva JC, Eckert KA, Resnikoff $\mathrm{S}$. The cataract situation in Latin America: Barriers to cataract surgery. Am J Ophthalmol . 2014;158(2):242-50.

6. El-Shafy E, El-Sayed S, El-Sobky H, Badawy $\mathrm{N}$. Phacoemulsification versus manual small incision cataract surgery for treatment of cataract. Menoufia Medical Journal. 2015;28(1):191.

7. Ruit $S$, Gurung R, Vyas $S$. The role of small incision suture-less cataract surgery in the developed world. Curr Opin Ophthalmol. 2018;29(1):105-9.
8. Singh SK, Winter I, Surin L. Phacoemulsification versus small incision cataract surgery (SICS): Which one is a better surgical option for immature cataract in developing countries?. Nepal J Ophthalmol. 2009;1(2):95-100.

9. Alada JJ, Umar MM, Alhassan MB, Mpyet C. Phacoemulsification: Practice and barriers in Nigeria. Niger J Ophthalmol. 2018;25(2):101-4.

10. Al-Muammar. Bimanual microincisional cataract surgery technique and clinical outcome. Saudi J Ophthalmol. 2009;23(2):149-55.

11. Zia SR, Syed IA. Comparison of small incision cataract surgery with extra capsular cataract extraction. J Rawal Med Coll. 2010;14(2):84-6.

12. Venkatesh R, Tan SHC, Sengupta S, Ravindran RD, Krishnan KT, Chang DF. Phacoemulsification versus manual small-incision cataract surgery for white cataract. J Cataract Refract Surg. 2010;36:1849-54.

13. Devendra J, Agarwal S, Singh P. A comparative study of clear corneal phacoemulsification with rigid IOL versus SICS; the preferred surgical technique in low socio-economic group patients of rural areas. J Clin Diagn Res. 2014;8(11):VC01-VC03.

14. Farmer L, Innes-Wong C, Bergman-Hart C, Casson RJ, Crompton J. Visual acuity, quality of life and visual function outcomes after cataract surgery in Bali. Ophthalmic Epidemiol. 2015;22(4):274-82.

15. Gogate P. Comparison of various techniques for cataract surgery, their efficacy, safety and cost. Oman J Ophthalmol. 2010;3(3):105-6.

16. Bhargava R, Kumar P, Bashir H, Sharma SK, Mishra A. Manual suture less small incision cataract surgery in patients with uveitic cataract. Middle East Afr J Ophthalmol. 2014;21(1):77-82. 